\title{
CODAZZI TENSOR FIELDS, CURVATURE AND PONTRYAGIN FORMS
}

\author{
ANDRZEJ DERDZIŃSKI and CHUN-LI SHEN
}

[Received 31 July 1982]

\section{Introduction}

A symmetric $(0,2)$ tensor field $b$ on a Riemannian manifold $(M, g)$ is said to be a Codazzi tensor if it satisfies the Codazzi equation

$$
\left(\nabla_{X} b\right)(Y, Z)=\left(\nabla_{Y} b\right)(X, Z)
$$

for arbitrary vector fields $X, Y, Z$. In this case, the self-adjoint section $B$ of End TM, characterized by $g(B X, Y)=b(X, Y)$, will also be called a Codazzi tensor. The Codazzi tensor $b$ will be called non-trivial if it is not a constant multiple of the metric.

The aim of the present paper is to study some geometric and topological consequences of the existence of a non-trivial Codazzi tensor on a given Riemannian manifold. Results of this type were obtained by Bourguignon [3], who proved that the existence of such a tensor imposes strong restrictions on the curvature operator [3, Théorème 5.1 and Corollaire 5.3] and, as a consequence, obtained the following theorem [3, Corollaire 7.3]: a compact orientable Riemannian four-manifold admitting a non-trivial Codazzi tensor with constant trace must have signature zero. Our main results consist in generalizing these theorems, in particular in seeing what can be said when the assumption on the trace is dropped.

In $\$ 2$ of this paper we observe that, in the $C^{\infty}$ category, every manifold admits a Riemannian metric with a non-trivial Codazzi tensor (Example 7), so that topological consequences may be expected only if some sort of analytic behaviour is assumed. Section 3 is devoted to the particular consequences of the existence of a non-trivial Codazzi tensor $B$ for the structure of the curvature operator (Theorem 1): for any point $x$ of the manifold $M$ and arbitrary eigenspaces $V_{\lambda}, V_{\mu}$ of $B_{x}$, the span $V_{\lambda} \wedge V_{\mu} \subset \Lambda^{2} T_{x} M$ of all exterior products of elements of $V_{\lambda}$ and $V_{\mu}$ is invariant under the curvature operator $R_{x}$ acting on 2-forms. As a consequence, we obtain in $\S 4$ a relation between the eigenspaces of any Codazzi tensor and the Pontryagin forms (Propositions 3 and 4), which, together with an extra argument for the case of a Codazzi tensor having only two distinct eigenvalues (Lemma 1), implies that a compact orientable Riemannian four-manifold $(M, g)$ admitting a non-trivial Codazzi tensor $b$ must have signature zero unless the restriction of b to some non-empty open subset of $M$ is a constant multiple of $g$ (Theorem 2). Another consequence of Proposition 4 is that for any n-dimensional Riemannian manifold with a Codazzi tensor having $n$ distinct eigenvalues almost everywhere, all the real Pontryagin classes are zero (Corollary 3 ).

The authors wish to express their gratitude to Professors Manfredo P. do Carmo, Hermann Karcher, and Udo Simon for valuable discussions and comments. 


\section{Examples of Codazzi tensors}

Codazzi tensors appear in a natural way in many geometric situations.

EXAMPLE 0. The simplest Codazzi tensors are parallel ones; non-trivial (i.e., not proportional to the metric) tensors of this type exist only in locally reducible manifolds.

EXAMPLE 1. For a space $(M, g)$ of constant sectional curvature $\kappa$ and any function $f$ on $M$, the formula $b=\nabla d f+\kappa f g$ defines a Codazzi tensor. As shown by Ferus [8], every Codazzi tensor in a space of constant curvature is, locally, of this type.

EXAMPLE 2. The second fundamental form of any hypersurface $(M, g)$ in a space of constant curvature is a Codazzi tensor (non-trivial, unless $M$ is totally umbilic).

EXAmple 3. Let $(M, g)$ be a conformally flat manifold, and let $n=\operatorname{dim} M \geqslant 3$. Then $b=$ Ric $-(2 n-2)^{-1}$ Scal. $g$ is a Codazzi tensor (non-trivial, unless $(M, g)$ is of constant curvature). In fact, for $n=3$, the Codazzi equation for $b$ is equivalent to the conformal flatness of $g$, while, for $n \geqslant 4$, the Weyl conformal tensor $W$ of any Riemannian $n$ manifold satisfies the well-known divergence formula

$$
(n-2) \nabla^{r} W_{r k i j}=(n-3)\left(\nabla_{i} b_{k j}-\nabla_{j} b_{k i}\right) .
$$

EXAMPLE 4. A Riemannian manifold is said to have harmonic curvature if $\delta R=0$ (in local coordinates, $\nabla^{r} R_{r k i j}=0$ ). This happens if and only if the Ricci tensor Ric satisfies the Codazzi equation. There exist various examples of compact manifolds with this property and with $\nabla$ Ric $\neq 0[7,5,13]$. In particular, such a metric always exists on the product $S^{1} \times N, N$ being any compact Einstein manifold of positive scalar curvature.

Example 5. Consider a Riemannian manifold $(M, g)$ admitting a function $f$ (not identically zero) such that

$$
\nabla d f=f\left[\text { Ric }-(n-1)^{-1} \text { Scal.g }\right], \quad n=\operatorname{dim} M .
$$

It is well known ([4, 9], cf. also [14]) that such $f$ exists if and only if the mapping assigning to metrics on $M$ their scalar curvature functions is not submersive at $g$. Moreover, (1) is necessary and sufficient in order that the metric $g+f^{2} \cdot d t^{2}$ on $\left(M \backslash f^{-1}(0)\right) \times S^{1}$ be Einsteinian. There are some obvious examples for (1) (the standard sphere $M=S^{n} \subset \mathbb{R}^{n+1}$, with $f$ linear; a Riemannian product $M=S^{1} \times N$, with $N$ an Einstein space of positive scalar curvature, $f$ being the composite of a linear function on $S^{1} \subset \mathbb{R}^{2}$ with the projection $S^{1} \times N \rightarrow S^{1}$ ). Moreover, as shown by Lafontaine [14], and, independently, by $\mathrm{O}$. Kobayashi, for any Einstein manifold $N$ of positive scalar curvature $(\operatorname{dim} N \geqslant 2)$, the product $S^{1} \times N$ admits a metric $g$ (essentially different from the obvious examples) such that (1) has a nontrivial solution $f$. If $n=3$ and $f$ satisfies (1) on $(M, g)$, then $b=f^{2}$ Ric $+\left(\frac{1}{2}|\nabla f|^{2}-\frac{1}{4}\right.$ Scal. $\left.f^{2}\right) g$ is a Codazzi tensor on $(M, g)$ (in fact, the Codazzi equation for $b$ is just the integrability condition for (1) with $n=3$ ).

Example 6. Fix a basis $X, Y, Z$ of left-invariant vector fields on the Lie group $S^{3} \simeq S U(2)$, satisfying the bracket relations $[X, Y]=Z,[Y, Z]=X,[Z, X]=Y$. 
Given a number $\gamma>0$ and mutually distinct real numbers $\lambda, \mu, v$, we define a leftinvariant metric $g$ and a $(1,1)$ tensor field $B$ on $S^{3}$ by

$$
\begin{gathered}
g(X, X)=\gamma(\mu-v)^{2}, \quad g(Y, Y)=\gamma(\lambda-v)^{2}, \quad g(Z, Z)=\gamma(\lambda-\mu)^{2}, \\
g(X, Y)=g(X, Z)=g(Y, Z)=0
\end{gathered}
$$

and $B X=\lambda X, B Y=\mu Y, B Z=v Z$. Then $B$ is a Codazzi tensor on $\left(S^{3}, g\right)$. Conversely, it is easy to show that if $(M, g)$ is a three-dimensional, complete, locally irreducible Riemannian manifold with a non-trivial Codazzi tensor $B$ having constant eigenvalues, then the universal covering of the triple $(M, g, B)$ is isometric to some $\left(S^{3}, g, B\right)$ of the type just described.

EXAMPLE 7. Every manifold $M$ carries a $C^{\infty}$ metric $g$ such that $(M, g)$ admits a nontrivial $C^{\infty}$ Codazzi tensor $b$. In fact, let $E$ be a subset of $M$, diffeomorphic to a closed ball in $\mathbb{R}^{n}$, where $n=\operatorname{dim} M$. Using a suitable embedding of $E$ in $\mathbb{R}^{n+1}$ (cf. Example 2), we can find a $C^{\infty}$ metric $g_{1}$ on $E$ and a non-trivial Codazzi tensor $b$ on $\left(E, g_{1}\right)$, vanishing near $\partial E$. Setting $b=0$ in $M \backslash E$, we may choose our $g$ to be any $C^{\infty}$ metric on $M$ such that $g=g_{1}$ wherever $b \neq 0$.

EXAmPLE 8. For Riemannian manifolds $\left(M_{i}, g_{i}\right)$, with $i=1,2$, and a function $f>0$ on $M_{1}$, one defines the warped product $(M, g)=\left(M_{1}, g_{1}\right) \times_{f}\left(M_{2}, g_{2}\right)$ by $M=M_{1} \times M_{2}$ and $g=\pi_{1}^{*} g_{1}+\left(f^{2} \circ \pi_{1}\right) \pi_{2}^{*} g_{2}, \pi_{i}: M \rightarrow M_{i}$ being the natural projection (cf. $\left.[2,12]\right)$. If $b^{\prime}$ is a Codazzi tensor of type $(0,2)$ on $\left(M_{2}, g_{2}\right)$, it is easy to verify that $b=\left(f \circ \pi_{1}\right) \pi_{2}^{*} b^{\prime}$ is a Codazzi tensor on $(M, g)$ (for the Riemannian connection of $g$, see [2, p. 24]).

Example 9. Let $(M, g, J)$ be a Kähler manifold. If $B$ is a Codazzi tensor of type $(1,1)$ on $(M, g)$ which is Hermitian with respect to $J$, that is, $B \circ J=J \circ B$, then $B$ is parallel. In fact, the $(1,1)$ tensor field $\zeta=J \circ B$ is skew-adjoint and hence so is $\nabla_{X} \zeta$ for any vector $X$; on the other hand, $\nabla J=0$ yields $\left(\nabla_{X} \zeta\right) Y=J\left(\left(\nabla_{X} B\right) Y\right)$ for arbitrary vectors $X, Y$. Thus, the expression $\left\langle\left(\nabla_{X} \zeta\right) Y, Z\right\rangle$ is symmetric in $X, Y$ and skew-symmetric in $Y, Z$ and therefore it must vanish identically (cf. [18]).

EXAmple 10. For a smooth manifold $M$ endowed with a linear connection $\nabla$, the $(1,1)$ tensor fields $B$ on $M$, satisfying the Codazzi equation $\left(\nabla_{X} B\right) Y=\left(\nabla_{Y} B\right) X$ for arbitrary vectors $X, Y$ can be called the Codazzi tensors on $(M, \nabla)$. They can be interpreted as follows:

(a) denoting by $d^{\nabla}$ the exterior differentiation operator on $T M$-valued forms in $M$, determined by $\nabla$, we see that a $T M$-valued 1 -form is a $\operatorname{Codazzi}$ tensor on $(M, \nabla)$ if and only if it is $d^{\nabla}$-closed (cf. [3]);

(b) considering a Codazzi tensor $B$ on $(M, \nabla)$ in the case where $M$ is compact, we may always assume that $B$ is non-degenerate everywhere, replacing it, if necessary, by $B+t$.Id for a sufficiently large $t$.

Any non-degenerate $(1,1)$ tensor field $B$ on $M$ is a vector bundle automorphism of $T M$, transforming $\nabla$ into the connection $\bar{\nabla}=B^{*} \nabla$ characterized by $B\left(\bar{\nabla}_{X} Y\right)=\nabla_{X}(B Y)$. The torsion tensors $T, \bar{T}$ of $\nabla$ and $\bar{\nabla}$ then satisfy the relation [10]

$$
B[\bar{T}(X, Y)-T(X, Y)]=\left(\nabla_{X} B\right) Y-\left(\nabla_{Y} B\right) X .
$$

Consequently, a section $B$ of $\mathrm{Aut}_{M}(T M)$ satisfies the Codazzi equation with respect to $\nabla$ if and only if $\nabla$ and $\bar{\nabla}=B^{*} \nabla$ have equal torsion tensors; since, in this case, $\nabla=\left(B^{-1}\right)^{*} \bar{\nabla}$, it follows that $B^{-1}$ satisfies the Codazzi equation with respect to $\bar{\nabla}$. 
From the above equality it also follows that a symmetric connection $\nabla$ on a compact manifold $M$ admits a Codazzi tensor which is not a constant multiple of Id if.and only if $\nabla$ can be transformed into some symmetric connection by an $M$-automorphism of TM not proportional to the identity.

It is clear that every Riemannian product carries non-trivial Codazzi tensors, which are parallel. However, by suitably deforming any product metric, one can obtain locally irreducible metrics on product manifolds, which still admit non-trivial Codazzi tensors.

Proposition 1. For arbitrary $C^{\infty}$ (respectively, analytic) manifolds $M_{1}, \ldots, M_{k}$, with $k \geqslant 1$, the product $M=M_{1} \times \ldots \times M_{k}$ carries a $C^{\infty}$ (respectively, analytic) locally irreducible Riemannian metric $g$ such that $(M, g)$ admits a $C^{\infty}$ (respectively analytic) Codazzi tensor $b$ which has precisely $k$ distinct eigenvalues at every point, all the eigenvalues being bounded on $M$, and whose eigenspace bundles coincide with the natural foliations of $M$ coming from the product structure. Moreover, the metric induced by such a $g$ on any integral manifold of an eigenspace foliation of $b$ can be prescribed arbitrarily (unless $k=1$, in which case it must be locally irreducible).

Proof. We proceed by induction on $k$. For $k=1$, our assertion is obvious. Suppose now that we have already found a metric $g^{\prime}$ with a Codazzi tensor $b^{\prime}$ on $M^{\prime}=M_{1} \times \ldots \times M_{k-1}$, having the required properties. Adding to $b^{\prime}$ a suitable constant multiple of $g^{\prime}$, we may assume that 0 is not an eigenvalue of $b^{\prime}$ at any point. For an arbitrary metric $g_{k}$ on $M_{k}$, we can clearly find a positive function $f$ on $M_{k}$, bounded away from zero and such that the warped product $(M, g)=\left(M_{k}, g_{k}\right) \times{ }_{f}\left(M^{\prime}, g^{\prime}\right)$ is locally irreducible. The Codazzi tensor $b$ on $(M, g)$, defined in terms of $b^{\prime}$ and $f$ as in Example 8, will then have $k$ distinct and uniformly bounded eigenvalues at each point, which completes the proof.

\section{Consequences for the curvature}

The following proposition, due to Hicks [10] (cf. also [19]), is the main step for proving our Theorem 1. It reduces all the arguments involving differentiations of a given Codazzi tensor to the mere fact that the curvature tensor of some new Riemannian metric has the generally valid symmetries, which makes the proof of Theorem 1 purely algebraic.

Proposition 2 (Hicks). Let $(M, g)$ be a Riemannian manifold, and let $B$ be a section of End $T M$, non-degenerate at each point. Define a new Riemannian metric $G$ and a linear connection $\bar{\nabla}$ on $M$ by $G(X, Y)=g(B X, B Y)$ and $\bar{\nabla}_{X} Y=\nabla_{X} Y+B^{-1}\left(\left(\nabla_{X} B\right) Y\right)$, that is, $B\left(\bar{\nabla}_{X} Y\right)=\nabla_{X}(B Y)$ for arbitrary vector fields $X, Y, \nabla$ being the Riemannian connection of $g$. Then

(i) $\bar{\nabla} G=0$ and the curvature tensor $\bar{R}$ of $\bar{\nabla}$ is characterized by

$$
G(\bar{R}(X, Y) Z, U)=R(X, Y, B Z, B U),
$$

(ii) if $B$ satisfies the Codazzi equation $\left(\nabla_{X} B\right) Y=\left(\nabla_{Y} B\right) X$, then $\bar{\nabla}$ is the Riemannian connection of $G$ and the curvature tensor $R^{G}$ (of type $\left.(0,4)\right)$ for $(M, G)$ is given by

$$
R^{G}(X, Y, Z, U)=R(X, Y, B Z, B U),
$$


(iii) if $B$ is a (self-adjoint) Codazzi tensor on $(M, g)$, then the symmetric tensor field $b$, defined by $b(X, Y)=g(B X, Y)$, is a Codazzi tensor on $(M, g)$ as well as on $(M, G)$.

Proof. $B$ is an automorphism (a 'gauge transformation') of the vector bundle $T M$ and our definitions of $G$ and $\bar{\nabla}$ can be written as $G=B^{*} g$ and $\bar{\nabla}=B^{*} \nabla$. Consequently, $\nabla G=0$ and $\bar{R}=B^{*} R$, which implies (i). If, moreover, $B$ is a Codazzi tensor on $(M, \nabla)$, then $\bar{\nabla}$ is torsion-free and $B^{-1}$ is a Codazzi tensor on $(M, \bar{\nabla})$ (cf. Example 10 ), which, together with the fact that $G\left(B^{-1} X, Y\right)=g(X, B Y)$, implies (ii) and (iii). This completes the proof.

Using Proposition 2, we can now prove our basic result on the structure of the curvature tensor $R$ of a Riemannian manifold $(M, g)$ admitting a non-trivial Codazzi tensor $B$. For any point $x \in M$ and eigenvalues $\lambda, \mu$ of $B_{x}$, we shall denote by $V_{\lambda}$, $V_{\mu} \subset T_{x} M$ the corresponding eigenspaces and by $V_{\lambda} \wedge V_{\mu} \subset \Lambda^{2} T_{x} M$ the subspace spanned by all exterior products $X \wedge Y$ with $X \in V_{\lambda}$ and $Y \in V_{\mu}$. The curvature tensor $R$ will be viewed, in the obvious way, as an operator acting on 2 -forms.

TheOrem 1. Let $B$ be a Codazzi tensor on a Riemannian manifold $(M, g)$. For any $x \in M$ and arbitrary eigenvalues $\lambda$, $\mu$ of $B_{x}$, the subspace $V_{\lambda} \wedge V_{\mu}$ of $\Lambda^{2} T_{x} M$ is invariant under the curvature operator $R_{x} \in$ End $\Lambda^{2} T_{x} M$. In other words, given eigenvalues $\lambda, \mu$, $v$ of $B_{x}$ and vectors $X \in V_{\lambda}, Y \in V_{\mu}, Z \in V_{v}$, we have

$$
R(X, Y) Z=0
$$

provided that $\lambda, \mu, v$ are mutually distinct or $\lambda=\mu \neq v$.

Proof. Replacing $B$ by $B+t$.Id for a suitable constant $t$, we may assume that $B$ is non-degenerate in a neighbourhood of $x$. Consider eigenvalues $\lambda, \mu, v, \xi$ of $B_{x}$ and vectors $X \in V_{\lambda}, Y \in V_{\mu}, Z \in V_{\nu}, U \in V_{\xi}$. Using (2), we obtain

$$
\nu \xi R(X, Y, Z, U)=R^{G}(X, Y, Z, U)=R^{G}(Z, U, X, Y)=\lambda \mu R(X, Y, Z, U)
$$

and, similarly, $(\mu \xi-\lambda v) R(X, Z, U, Y)=(\mu \nu-\lambda \xi) R(X, U, Y, Z)=0$. In view of (2), we also have

$$
\begin{aligned}
0 & =R^{G}(X, Y, Z, U)+R^{G}(X, Z, U, Y)+R^{G}(X, U, Y, Z) \\
& =v \xi R(X, Y, Z, U)+\mu \xi R(X, Z, U, Y)+\mu \nu R(X, U, Y, Z),
\end{aligned}
$$

so that the preceding equalities, together with $\mu \nu \xi \neq 0$, yield the matrix equation

$$
\left[\begin{array}{lll}
\lambda & \xi & v \\
\xi & \lambda & \mu \\
\nu & \mu & \lambda \\
1 & 1 & 1
\end{array}\right] \cdot\left[\begin{array}{c}
R(X, Y, Z, U) \\
R(X, Z, U, Y) \\
R(X, U, Y, Z)
\end{array}\right]=\left[\begin{array}{l}
0 \\
0 \\
0 \\
0
\end{array}\right]
$$

Suppose that $R(X, Y, Z, U) \neq 0$. Then, the coefficient matrix above is of rank at most 2 , which implies the cofactor relations

$$
(\lambda-\xi)(\lambda+\xi-\mu-v)=(v-\lambda)(v+\lambda-\mu-\xi)=(\lambda-\mu)(\lambda+\mu-v-\xi)=0 .
$$


If we now had $\lambda \neq \mu, \lambda \neq v$, and $\lambda \neq \xi$, these relations would give a contradiction $(\lambda=\mu=\nu=\xi)$. Consequently, $R(X, Y, Z, U)$ can be non-zero only if $\lambda$ is equal to one of $\mu, v, \xi$. The symmetries of $R$ imply now that, for arbitrary eigenvectors $X_{1}, \ldots, X_{4}$ of $B_{x}, R\left(X_{1}, X_{2}, X_{3}, X_{4}\right)$ can be non-zero only if $X_{1}, \ldots, X_{4}$ belong to at most two distinct eigenspaces. On the other hand, if $X, Y \in V_{\lambda}, Z \in V_{v}$, and $\lambda \neq v$, (2) yields

$$
\begin{aligned}
0 & =B\left[R^{G}(X, Y) Z+R^{G}(Y, Z) X+R^{G}(Z, X) Y\right] \\
& =R(X, Y)(B Z)+R(Y, Z)(B X)+R(Z, X)(B Y) \\
& =(v-\lambda) R(X, Y) Z .
\end{aligned}
$$

This completes the proof.

Any two self-adjoint $(1,1)$ tensor fields $A, B$ on a Riemannian manifold $(M, g)$ give rise to a section $A \otimes B$ of End $\Lambda^{2} T M$ defined by

$$
(A \otimes B)(X \wedge Y)=\frac{1}{2}(A X \wedge B Y+B X \wedge A Y) .
$$

Thus, (2) can be rewritten as $R^{G}=(B \otimes B) \circ R$, where $R^{G}$ is viewed as a section of End $\Lambda^{2} T M$ with the aid of $g$. The Weyl conformal tensor $W$ of $(M, g)$ acting on 2forms, is given by

$$
W=R-2(n-2)^{-1} \mathrm{Id} \otimes \operatorname{Ric}+(n-1)^{-1}(n-2)^{-1} \text { Scal } \text { Id } \otimes \mathrm{Id}, \quad \text { where } n=\operatorname{dim} M \text {. }
$$

If $x \in M$ and $V_{\lambda}, V_{\mu} \subset T_{x} M$ are the eigenspaces corresponding to the eigenvalues $\lambda, \mu$ of $B_{x}$, then the restriction of $(\operatorname{Id} \otimes B)_{x}$ to $V_{\lambda} \wedge V_{\mu}$ equals $\frac{1}{2}(\lambda+\mu)$ times the identity. Suppose now that $B$ is a Codazzi tensor. If $X \in V_{\lambda}$ and $Y \in V_{\mu}$, where $\lambda, \mu$ are distinct eigenvalues of $B_{x}$, then Theorem 1 yields $R(X, Z, Y, Z)=0$ for any eigenvector $Z$ of $B_{x}$. Therefore $\operatorname{Ric}(X, Y)=0$. Hence the Ricci tensor commutes with $B$ and, since $\Lambda^{2} T_{x} M$ is spanned by all the $V_{\lambda} \wedge V_{\mu}$, Theorem 1 implies that the endomorphisms $R$, Id $\otimes$ Ric, and $W$ of $\Lambda^{2} T M$ commute with Id $\otimes B$. Consequently, we obtain the following commutation theorem, due to Bourguignon [3, Théorème 5.1]; note that this result implies our Theorem 1 except for the case where $B_{x}$ has two distinct pairs $\{\lambda, \mu\},\{\nu, \xi\}$ of eigenvalues with $\lambda+\mu=\nu+\xi$ : in this case, $V_{\lambda} \wedge V_{\mu}$ and $V_{v} \wedge V_{\xi}$ are proper subspaces of some eigenspace of (Id $\otimes B)_{x}$ and so their $R_{x}$-invariance is not an algebraic consequence of the fact that the endomorphisms commute.

COROLlaRY 1 (Bourguignon). Let $B$ be a Codazzi tensor on a Riemannian manifold $(M, g)$. Then

(i) B commutes with the Ricci tensor Ric,

(ii) the endomorphisms $R$, Id $\otimes$ Ric, and $W$ of $\Lambda^{2} T M$ commute with Id $\otimes B$.

Let us now consider a non-trivial Codazzi tensor $B$ on a Riemannian manifold $(M, g)$. Given a point $x \in M$, it is natural to study the components of the curvature tensor $R_{x}$ with respect to an orthonormal basis of eigenvectors of $B_{x}$. According to Theorem 1, there are, essentially, only two types of these components, which may be non-zero: they have the form $R(X, Y, Z, U)$ with $X, Z \in V_{\lambda}$ and $Y, U \in V_{\mu}, \lambda, \mu$ being eigenvalues of $B_{x}$ such that either

$$
i=\mu,
$$

or

$$
i \neq \mu \text {. }
$$


The obvious question that arises is whether the Codazzi equation for $B$ imposes any algebraic restrictions on these components, which are not consequences of the general curvature symmetries. For the components of Type (I), the answer is negative, the simplest examples being the parallel tensors on Riemannian product manifolds; moreover, the same situation occurs when the underlying manifold is assumed locally irreducible. In fact, restricting our consideration to the connected components of some open dense subset of $M$, we may view the eigenvalues of the given self-adjoint $(1,1)$ tensor field $B$ as smooth functions, while the eigenspaces of $B$ form differentiable distributions. If $B$ is a Codazzi tensor, these distributions must be integrable and their leaves are totally umbilic in $M([7$, Lemma 2$]$; more general results can be found in [11]). The curvature components of Type (I) are, thus, closely related to the intrinsic curvatures of the leaves, which, by Proposition 1, may be completely arbitrary also in the locally irreducible case. On the other hand, for a Riemannian manifold with a non-trivial Codazzi tensor, the curvature components of Type (II) must have some special algebraic properties, at least in the case of a Codazzi tensor with only two distinct eigenvalues. Namely, we have

Lemma 1. Let $(M, g)$ be a Riemannian manifold with a Codazzi tensor $B$ which has, at each point $x \in M$, exactly two distinct eigenvalues $\lambda(x), \mu(x)$, with $\lambda(x)<\mu(x)$. This gives rise to smooth eigenvalue functions $\lambda, \mu$ and eigenspace distributions $V_{\lambda}, V_{\mu}$ on $M$. For any point $x \in M$ and arbitrary eigenvectors $X, Z \in V_{\lambda}(x), Y, U \in V_{\mu}(x)$, we have

$$
\begin{aligned}
(\lambda-\mu)^{2} R(X, Y, Z, U)= & -g(\nabla \lambda, \nabla \mu) g(X, Z) g(Y, U) \\
& +g(X, Z) A_{\mu}(Y, U)+A_{\lambda}(X, Z) g(Y, U),
\end{aligned}
$$

$A_{\lambda}$ (respectively, $A_{\mu}$ ) being the symmetric bilinear form on $V_{\lambda}$ (respectively, on $\left.V_{\mu}\right)$, obtained by restricting the bilinear form $(\mu-\lambda) \nabla d \mu-2 d \mu \otimes d \mu+d \lambda \otimes d \mu$ to $V_{\lambda}$ (respectively, by restricting $(\lambda-\mu) \nabla d \lambda-2 d \lambda \otimes d \lambda+d \mu \otimes d \lambda$ to $V_{\mu}$ ). Consequently, there exist orthonormal bases $e_{1}, \ldots, e_{m}$ of $V_{\lambda}(x), e_{m+1}, \ldots, e_{n}$ of $V_{\mu}(x)$ (where $\left.m=\operatorname{dim} V_{\lambda}, n=\operatorname{dim} M\right)$ such that, for certain real numbers $c_{1}, \ldots, c_{n}$,

$$
R\left(e_{i}, e_{a}, e_{j}, e_{b}\right)=\left(c_{i}+c_{a}\right) \delta_{i j} \delta_{a b}
$$

whenever $1 \leqslant i, j \leqslant m<a, b \leqslant n$.

Proof. The vector bundle decomposition $T M=V_{\lambda} \oplus V_{\mu}$ together with the Riemannian connection $\nabla$ in $T M$ gives rise to the connections $\nabla^{\lambda}, \nabla^{\mu}$ in $V_{\lambda}, V_{\mu}$, respectively, defined in the obvious way. On the other hand, for arbitrary local sections $X, Z$ of $V_{\lambda}$ and $Y, U$ of $V_{\mu}$, we have

$$
(\lambda-\mu) g\left(\nabla_{X} Z, Y\right)=g(X, Z) \nabla_{Y} \lambda
$$

(by Lemma 2 of [7], $V_{\lambda}$ is integrable, so that both sides of this equality are symmetric in $X, Z$, while the equality holds for $X=Z$ by (ii) of Lemma 1 of [7]), and, similarly, $(\mu-\lambda) g\left(\nabla_{Y} U, X\right)=g(Y, U) \nabla_{X} \mu$. Consequently, for $X, Y, Z, U$ as above,

$$
\begin{aligned}
& \nabla_{X} Z=\nabla_{X}^{\lambda} Z+(\lambda-\mu)^{-1} g(X, Z)(\nabla \lambda)_{V_{\mu}}, \\
& \nabla_{X} Y=(\mu-\lambda)^{-1} \nabla_{Y} \lambda . X+\nabla_{X}^{\mu} Y, \\
& \nabla_{Y} X=(\lambda-\mu)^{-1} \nabla_{X} \mu . Y+\nabla_{Y}^{\lambda} X, \\
& \nabla_{Y} U=\nabla_{Y}^{\mu} U+(\mu-\lambda)^{-1} g(Y, U)(\nabla \mu)_{V_{\lambda}},
\end{aligned}
$$


$(\nabla \lambda)_{V_{\mu}}$ being the $V_{\mu}$ component of $\nabla \lambda$. This implies

$$
\begin{aligned}
g\left(U, \nabla_{Y} \nabla_{X} Z\right)=(\lambda-\mu)^{-1} g\left(\nabla \mu, \nabla_{X}^{\lambda} Z\right) g(U, Y)+(\lambda-\mu)^{-2} \nabla_{Y}(\mu-\lambda) \cdot g(X, Z) \nabla_{U} \lambda \\
+(\lambda-\mu)^{-1}\left[g\left(\nabla_{Y}^{\lambda} X, Z\right)+g\left(X, \nabla_{Y}^{\lambda} Z\right)\right] \nabla_{U} \lambda \\
+(\lambda-\mu)^{-1} g\left(\nabla_{Y}(\nabla \lambda)_{V_{\mu}}, U\right) g(X, Z)
\end{aligned}
$$

and

$$
\begin{aligned}
-g\left(U, \nabla_{X} \nabla_{Y} Z\right)= & (\lambda-\mu)^{-2} \nabla_{X}(\lambda-\mu) \cdot g(Y, U) \nabla_{Z} \mu \\
& -(\lambda-\mu)^{-1} \nabla_{X} \nabla_{Z} \mu \cdot g(Y, U)-(\lambda-\mu)^{-1} g\left(\nabla_{X}^{\mu} Y, U\right) \nabla_{Z} \mu \\
& -(\lambda-\mu)^{-1} g\left(X, \nabla_{Y}^{\lambda} Z\right) \nabla_{U} \lambda,
\end{aligned}
$$

and

$$
\begin{aligned}
g\left(U, \nabla_{[X, Y]} Z\right)= & (\lambda-\mu)^{-1} g\left(\nabla_{X}^{\mu} Y, U\right) \nabla_{Z} \mu-(\lambda-\mu)^{-1} g\left(\nabla_{Y}^{\lambda} X, Z\right) \nabla_{U} \lambda \\
& -(\lambda-\mu)^{-2} g(X, Z) \nabla_{Y} \lambda . \nabla_{U} \lambda-(\lambda-\mu)^{-2} \nabla_{X} \mu . \nabla_{Z} \mu . g(Y, U) .
\end{aligned}
$$

Adding up the last relations and using the obvious equalities

$$
\begin{aligned}
g\left(\nabla_{Y}(\nabla \lambda)_{V_{\mu}}, U\right) & =g\left(\nabla_{Y} \nabla \lambda, U\right)-g\left(\nabla_{Y}(\nabla \lambda)_{V_{\lambda}}, U\right) \\
& =(\nabla d \lambda)(U, Y)+(\mu-\lambda)^{-1} g\left((\nabla \lambda)_{V_{\lambda}}, \nabla \mu\right) g(U, Y), \\
\nabla_{X} \nabla_{Z} \mu & =g\left(\nabla_{X}(\nabla \mu), Z\right)+g\left(\nabla_{X} Z, \nabla \mu\right) \\
& =(\nabla d \mu)(X, Z)+g\left(\nabla_{X}^{\lambda} Z, \nabla \mu\right)+(\lambda-\mu)^{-1} g(X, Z) g\left((\nabla \lambda)_{V_{\mu}}, \nabla \mu\right)
\end{aligned}
$$

and

$$
\nabla \lambda=(\nabla \lambda)_{V_{\lambda}}+(\nabla \lambda)_{V_{\mu}},
$$

we obtain (3). The symmetry of $A_{\lambda}$ and $A_{\mu}$, immediate from the Bianchi identity for $R$ together with Theorem 1, can also be verified as follows: the only term of $A_{\lambda}$ which is not obviously symmetric is the restriction of $d \lambda \otimes d \mu$ to $V_{\lambda}$; however, by Lemma 2 of [7], $\lambda$ is constant along $V_{\lambda}$ unless $\operatorname{dim} V_{\lambda}=1$. Finally, (4) can be obtained by diagonalizing $A_{\lambda}$ and $A_{\mu}$, which completes the proof.

Remark 1. Assuming in Lemma 1 that $\operatorname{dim} V_{\lambda} \geqslant 2$ and $\operatorname{dim} V_{\mu} \geqslant 2$, we can reduce formula (3) to the simpler form

$$
\begin{aligned}
\varphi R(X, Y, Z, U)= & -g(X, Z) \nabla d \varphi(Y, U)-\nabla d \varphi(X, Z) g(Y, U) \\
& +\varphi^{-1}|\nabla \varphi|^{2} g(X, Z) g(Y, U)
\end{aligned}
$$

for $X, Y \in V_{\lambda}$ and $Y, U \in V_{\mu}$, where $\varphi=(\lambda-\mu)^{-1}$. In fact, the constancy of $\lambda$ and $\mu$ along $V_{\lambda}$ and $V_{\mu}$, respectively [7, Lemma 2] yields $g(\nabla \lambda, \nabla \mu)=0$ and

$$
A_{\lambda}(X, Z)=-\varphi^{-3} \nabla d \varphi(X, Z)+(\mu-\lambda) \nabla d \lambda(X, Z),
$$

while

$$
\nabla d \lambda(X, Z)=-d \lambda\left(\nabla_{X} Z\right)=-d \lambda\left(\left(\nabla_{X} Z\right)_{V_{\mu}}\right) .
$$

Since $(\lambda-\mu)\left(\nabla_{X} Z\right)_{V_{\mu}}=g(X, Z) \nabla \lambda$ (cf. the first equality in the proof of Lemma 1), we obtain

$$
A_{\lambda}(X, Z)=-\varphi^{-3} \nabla d \varphi(X, Z)+|\nabla \lambda|^{2} g(X, Z)
$$

and, similarly,

$$
A_{\mu}(Y, U)=-\varphi^{-3} \nabla d \varphi(Y, U)+|\nabla \mu|^{2} g(Y, U),
$$

so that our assertion is immediate from (3). 


\section{Codazzi tensors and Pontryagin forms}

Given a Riemannian manifold $(M, g)$ and a positive integer $k$, one defines the $k$ th Pontryagin form $P_{k}=P_{k}(M, g)$ of $(M, g)$, which is a closed $4 k$-form on $M$, obtained, locally, by applying some standard homogeneous polynomials of degree $2 k$ to the local curvature components [17, p. 308] and represents, in the de Rham cohomology of $M$, the real Pontryagin class $p_{k} \in H^{4 k}(M, \mathbb{R})$. By the Pontryagin algebra of $(M, g)$ we shall mean the graded subalgebra of $\Gamma(\Lambda M)$ (sections of $\Lambda M=\Lambda^{0} T^{*} M+\ldots+\Lambda^{n} T^{*} M$, where $n=\operatorname{dim} M$, with pointwise exterior multiplication), generated by all the $P_{k}$. It is sometimes more convenient to consider, instead of $P_{k}$, another system $\Omega_{k}(k=1,2, \ldots)$ of generators for the Pontryagin algebra of $(M, g)$, described as follows: $\Omega_{k}$ is the $4 k$-form on $M$, obtained by alternating (taking the skew-symmetric part of) the tensor field $\omega_{k}$ of type $(0,4 k)$ on $M$, given by

$$
\omega_{k}\left(X_{1}, \ldots, X_{4 k}\right)=\operatorname{Trace}\left[R\left(X_{1} \wedge X_{2}\right) \circ R\left(X_{3} \wedge X_{4}\right) \circ \ldots \circ R\left(X_{4 k-1} \wedge X_{4 k}\right)\right],
$$

the curvature tensor $R$ of $(M, g)$ being viewed as a homomorphism $\Lambda^{2} T M \rightarrow$ End $T M$. Every Pontryagin form can be obtained by applying some universal polynomial to the $\Omega_{k}$.

Consider now a finite-dimensional real vector space $T$. A fixed inner product $\langle$, allows us to identify $T$ with its dual $T^{*}, T \otimes T$ with $T \otimes T^{*}=$ End $T$, and $\Lambda^{2} T$ with a subspace of End $T$. Thus, for vectors $X, Y, Z, U \in T$, we have

$$
\operatorname{Trace}(X \otimes Y)=\langle X, Y\rangle
$$

and

$$
X \wedge Y=X \otimes Y-Y \otimes X
$$$$
(X \otimes Y) \circ(Z \otimes U)=\langle Y, Z\rangle . X \otimes U \text {. }
$$

For subspaces $V, V^{\prime}$ of $T$, we define $V \otimes V^{\prime} \subset T \otimes T$ to be the span of all tensor products $X \otimes X^{\prime}$ with $X \in V, X^{\prime} \in V^{\prime}$. In an analogous way, we define $V \wedge V^{\prime} \subset \Lambda^{2} T$ and, for subspaces $P, P^{\prime}$ of End $T$, the subspaces $P \circ P^{\prime}$ and $P+P^{\prime}$.

Lemma 2. Suppose we are given a finite-dimensional real vector space $T$ with an inner product, a subspace $V$ of $T$, and an endomorphism $R$ of $\Lambda^{2} T$ leaving the subspaces $V \wedge V, V \wedge V^{\perp}$, and $V^{\perp} \wedge V^{\perp}$ invariant. For any sequence $X_{1}, \ldots, X_{2 r}(r \geqslant 1)$ of vectors, containing exactly $m$ elements of $V$ and $2 r-m$ elements of $V^{\perp}$, we have

(i) $R\left(X_{1} \wedge X_{2}\right) \circ R\left(X_{3} \wedge X_{4}\right) \circ \ldots \circ R\left(X_{2 r-1} \wedge X_{2 r}\right)$

$$
\in \begin{cases}(V \otimes V)+\left(V^{\perp} \otimes V^{\perp}\right), & \text { if } m \text { is even, } \\ \left(V \otimes V^{\perp}\right)+\left(V^{\perp} \otimes V\right), & \text { if } m \text { is odd, }\end{cases}
$$

(ii) Trace $\left[R\left(X_{1} \wedge X_{2}\right) \circ \ldots \circ R\left(X_{2 r-1} \wedge X_{2 r}\right)\right]=0$ if $m$ is odd.

Proof. Set $V_{1}=V, V_{-1}=V^{\perp}$, and

$$
P(1)=(V \otimes V)+\left(V^{\perp} \otimes V^{\perp}\right), \quad P(-1)=\left(V \otimes V^{\perp}\right)+\left(V^{\perp} \otimes V\right) .
$$

By (7) and our hypothesis on $R, P(\delta) \circ P(\varepsilon) \subset P(\delta \varepsilon)$ and $R\left(V_{\delta} \wedge V_{\varepsilon}\right) \subset V_{\delta} \wedge V_{\varepsilon} \subset P(\delta \varepsilon)$ whenever $\delta, \varepsilon= \pm 1$. Therefore, if $X_{i} \in V_{\delta_{i}}$, for $i=1, \ldots, 2 r$, then

$$
R\left(X_{1} \wedge X_{2}\right) \circ \ldots \circ R\left(X_{2 r-1} \wedge X_{2 r}\right) \in P\left(\delta_{1} \delta_{2} \ldots \delta_{2 r}\right),
$$

which proves (i). Assertion (ii) is now immediate from (6), which completes the proof. 
As an immediate consequence of Theorem 1 and Lemma 2, we obtain

Proposition 3. Let $(M, g)$ be a Riemannian manifold with a Codazzi tensor $B$, let $x$ be a point of $M$, and let $V_{\lambda} \subset T_{x} M$ be the eigenspace corresponding to an eigenvalue $\lambda$ of $B_{x}$. Given a positive integer $k$, an odd integer $m(0 \leqslant m \leqslant 4 k)$, and arbitrary vectors $X_{1}, \ldots, X_{4 k} \in T_{x} M$ such that $X_{1}, \ldots, X_{m} \in V_{\lambda}$ and $X_{m+1}, \ldots, X_{4 k} \in V_{\lambda}^{\perp}$, every element $\Omega$ of degree $4 k$ in the Pontryagin algebra of $(M, g)$ satisfies the relation

$$
\Omega\left(X_{1}, \ldots, X_{4 k}\right)=0 \text {. }
$$

In particular, if $\lambda$ is a simple eigenvalue of $B_{x}$ and $X \in V_{\lambda}$, then

$$
l_{X} P_{k}=P_{k}(X, \cdot, \ldots, \cdot)=0
$$

for each Pontryagin form $P_{k}$, with $k \geqslant 1$.

Proof. In view of Theorem 1, we can apply Lemma 2 to $T=T_{x} M$ and $V=V_{\lambda}$. Consequently, the tensor $\omega_{k}$ given by (5) has the property that $\omega_{k}\left(X_{1}, \ldots, X_{4 k}\right)=0$ whenever, for some odd integer $m$, the sequence $X_{1}, \ldots, X_{4 k}$ contains $m$ elements of $V_{\lambda}$ and $4 k-m$ elements of $V_{\lambda}^{\perp}$. The same must hold for the alternation $\Omega_{k}$ of $\omega_{k}$ and hence for all forms in the Pontryagin algebra of $(M, g)$, which completes the proof.

From Proposition 3 we now obtain the following sufficient conditions for the vanishing of certain Pontryagin forms.

Proposition 4. Let $(M, g)$ be a Riemannian manifold with a Codazzi tensor $B$, and let $x$ be a point of $M$. If $B_{x}$ has exactly $p$ distinct eigenvalues $\lambda_{1}, \ldots, \lambda_{p}$ of multiplicities $m_{1}, \ldots, m_{p}$, respectively, then every homogeneous element of degree greater than

$$
2 \sum_{i=1}^{p}\left[\frac{1}{2} m_{i}\right]
$$

in the Pontryagin algebra of $(M, g)$ vanishes at $x,\left[\frac{1}{2} m_{i}\right]$ being the integer part of $\frac{1}{2} m_{i}$.

Proof. Let $\Omega$ be a homogeneous element of degree $4 r$ in the Pontryagin algebra of $(M, g)$ such that $\Omega_{x} \neq 0$. Thus, $\Omega\left(X_{1}, \ldots, X_{4 r}\right) \neq 0$ for some sequence $X_{1}, \ldots, X_{4 r}$ of linearly independent eigenvectors of $B_{x}$. By Proposition 3, for any eigenvalue $\lambda_{i}$ of $B_{x}$, the number of times that eigenvectors corresponding to $\lambda_{i}$ occur among the $X_{1}, \ldots, X_{4 r}$ is even and not greater than the multiplicity $m_{i}$, that is, it does not exceed $2\left[\frac{1}{2} m_{i}\right]$. Consequently, $\Omega_{x} \neq 0$ implies that $\operatorname{deg} \Omega=4 r \leqslant 2 \sum_{i=1}^{p}\left[\frac{1}{2} m_{i}\right]$, which completes the proof.

In particular, we have

Corollary 2. Let $(M, g)$ be an n-dimensional Riemannian manifold with a Codazzi tensor $B$, and let $x$ be a point of $M$. If $B_{x}$ has exactly $k$ simple eigenvalues, where $0 \leqslant k \leqslant n$, then every homogeneous element of degree greater than $n-k$ in the Pontryagin algebra of $(M, g)$ vanishes at $x$.

Proof. Let $m_{1} \leqslant \ldots \leqslant m_{p}$ be the multiplicities of the distinct eigenvalues of $B_{x}$, so that $m_{1}=\ldots=m_{k}=1$. Our assertion follows now from Proposition 4 together with 
the fact that

$$
n-k=\sum_{i>k} m_{i} \geqslant 2 \sum_{i>k}\left[\frac{1}{2} m_{i}\right]=2 \sum_{i=1}^{p}\left[\frac{1}{2} m_{i}\right]
$$

COROllaRy 3. Let $B$ be a Codazzi tensor on an n-dimensional Riemannian manifold $(M, g)$. If, for some $x \in M, B_{x}$ has $n$ distinct eigenvalues, then all the Pontryagin forms of $(M, g)$ vanish at $x$.

REMARK 2. It seems useful to consider the following concept, introduced by Maillot $[15,16]$ : a Riemannian manifold $(M, g)$ is said to have pure curvature operator at a point $x \in M$ if, for some orthonormal basis $X_{1}, \ldots, X_{n}$ of $T_{x} M$, each exterior product $X_{i} \wedge X_{j}(1 \leqslant i<j \leqslant n=\operatorname{dim} M)$ is an eigenvector of $R_{x} \in$ End $\Lambda^{2} T_{x} M$. This happens, for example, if $R_{x}=\sum_{\alpha} A_{\alpha} \otimes B_{\alpha}$ with mutually commuting self-adjoint $(1,1)$ tensors $A_{\alpha}, B_{\alpha}$, so that the simplest examples where the curvature operator is pure at each point are provided by conformally flat manifolds and by submanifolds of space forms having flat normal connection. As observed by Maillot, pure curvature operator at a point $x$ implies that all the Pontryagin forms vanish at $x$ (this is also immediate from Lemma 2 applied to $V=\operatorname{span}\left(X_{i}\right)$, for $\left.i=1, \ldots, n\right)$. By Theorem 1 , if $B$ is a Codazzi tensor on an $n$-dimensional Riemannian manifold $(M, g)$ and, for some point $x, B_{x}$ has $n$ distinct eigenvalues, then $(M, g)$ has pure curvature operator at $x$ (cf. Corollary 3).

For Codazzi tensors on four-dimensional manifolds, the preceding results imply the following theorem.

THEOREM 2. Let $B$ be a Codazzi tensor on a four-dimensional Riemannian manifold $(M, g)$. The Pontryagin form $P_{1}$ of $(M, g)$ then satisfies the relation

$$
P_{1} \otimes\left(B-\frac{1}{4} \text { Trace } B . \mathrm{Id}\right)=0 \text {. }
$$

Proof. We shall prove that $P_{1}(x)=0$ at all points $x$ at which the number of eigenvalues of $B$ is locally constant and greater than 1 ; the set of these points is dense in the subset of $M$ defined by $B \neq \frac{1}{4}$ Trace $B$. Id. For such an $x$, the number of eigenvalues of $B_{x}$ may equal 2 (multiplicities: $\{1,3\}$ or $\{2,2\}$ ), 3 (multiplicities: $\{1,1,2\}$ ), or 4 (multiplicities: $\{1,1,1,1\}$ ). By Corollary 2, $P_{1}(x)=0$ unless the distribution of multiplicities is $\{2,2\}$. However, in the latter case formula (4), together with Theorem 1 , implies that $(M, g)$ has pure curvature operator, which again yields $P_{1}(x)=0$ (Remark 2). This completes the proof.

In the case of compact oriented four-manifolds, Theorem 2, together with Hirzebruch's signature formula $3 \tau(M)=\int_{M} P_{1}$, yields

Corollary 4. Let $(M, g)$ be a compact, orientable, analytic four-dimensional Riemannian manifold admitting a non-trivial analytic Codazzi tensor. Then, the signature of $M$ is zero.

An oriented Riemannian four-manifold $(M, g)$ is called self-dual [1] if $W \circ *=W$, the Weyl tensor $W$ and the Hodge star $*$ being viewed as endomorphisms of $\Lambda^{2} T M$. For any compact self-dual manifold $(M, g)$, the signature $\tau(M) \geqslant 0$; the inequality being strict unless $W=0$ identically (see [1]). On the other hand, one can define the 
divergence $\delta W$ of $W$ by the local coordinate formula $(\delta W)_{i j k}=-\nabla^{r} W_{r i j k}$. Corollary 4 now gives a new proof of the following result [6, Proposition $7 ; 3$, Proposition 9.1].

COROllary 5. Let $(M, g)$ be a compact, analytic, oriented Riemannian fourmanifold. If $(M, g)$ is self-dual and $\delta W=0$, then $(M, g)$ is conformally flat or Einsteinian.

Proof. The condition $\delta W=0$ means that $b=$ Ric $-\frac{1}{6}$ Scal. $g$ is a Codazzi tensor (Example 3). If $\tau(M)=0$, then $W=0$ in view of self-duality. On the other hand, if $\tau(M) \neq 0$, Corollary 4 implies that $b$ is a multiple of $g$, that is, $g$ is an Einstein metric, which completes the proof.

\section{References}

1. M. F. Atiyah, N. J. Hitchin, and I. M. Singer, 'Self-duality in four-dimensional Riemannian geometry', Proc. Roy. Soc. London Ser. A, 362 (1978), 425-461.

2. R. L. Bishop and B. O'Neill, 'Manifolds of negative curvature', Trans. Amer. Math. Soc., 145 (1969), $1-49$.

3. J. P. BouRguignon, 'Les variétés de dimension 4 à signature non nulle dont la courbure est harmonique sont d'Einstein', Invent. Math., 63 (1981), 263-286.

4. J. P. Bourguignon, 'Une stratification de l'espace des structures riemanniennes', Compositio Math., 30 (1975), 1-41.

5. A. DERDZIŃSKI, 'On compact Riemannian manifolds with harmonic curvature', Math. Ann., 259 (1982), $145-152$.

6. A. DERDZIŃSKI, 'Self-dual Kähler manifolds and Einstein manifolds of dimension four', Compositio Math., to appear.

7. A. DERDZIŃSKI, 'Classification of certain compact Riemannian manifolds with harmonic curvature and non-parallel Ricci tensor', Math. Z., 172 (1980), 273-280.

8. D. FERUS, 'A remark on Codazzi tensors in constant curvature spaces', Global differential geometry and global analysis, Lecture Notes in Mathematics 838 (Springer, Berlin, 1981), p. 257.

9. A. E. FisCher and J. E. MARSDEN, 'Manifolds of Riemannian metrics with prescribed Ricci curvature', Bull. Amer. Math. Soc., 80 (1974), 479-484.

10. N. Hicks, 'Linear perturbations of connexions', Michigan Math. J., 12 (1965), 389-397.

11. S. HIEPKO and H. ReCKZIEGEL, 'Über sphärische Blätterungen und die Vollständigkeit ihrer Blätter', Manuscripta Math., 31 (1980), 269-283.

12. G. I. KruČKovıČ, 'On semi-reducible Riemannian spaces' (in Russian), Dokl. Akad. Nauk SSSR, 115 (1957), 862-865; MR 20\#2751.

13. J. Lafontaine, 'Remarques sur les variétés conformèment plates', Math. Ann., to appear.

14. J. Lafontaine, 'Sur la géométrie d'une généralisation de l'équation différentielle d'Obata', J. Math. Pures Appl., to appear.

15. H. Maillot, 'Sur les variétés riemanniennes à opérateur de courbure pur', C. R. Acad. Sci. Paris Sér. A, 278 (1974), 1127-1130.

16. H. Malloor, 'Sur l'opérateur de courbure d'une variété riemannienne', Thèse (3ème cycle), Université Claude-Bernard, Lyon, 1974.

17. J. W. MiLnor and J. D. StaShefF, Characteristic classes (Princeton University Press, Princeton, 1974).

18. S. Tanno, 'Curvature tensors and covariant derivatives', Ann. Mat. Pura Appl., 96 (1973), 233-241.

19. B. WEGNER, 'Codazzi-Tensoren und Kennzeichnungen sphärischer Immersionen', J. Differential Geom., 9 (1974), 61-70.

Present address:

SFB 40, Universität Bonn

Beringstrasse 4

5300 Bonn 1

Federal Republic of Germany
Department of Mathematics

Fudan University

Shanghai

People's Republic of China 